\title{
Editorial
}

\section{Current Concepts and Recent Developments in Shoulder Surgery}

\author{
Robert J. MacFarlane ${ }^{\S}$ and Mohammad Waseem ${ }^{*} \S$
}

\author{
Department of Trauma and Orthopaedics, Macclesfield District General Hospital, East Cheshire NHS Trust, Victoria \\ Road, Macclesfield, SK10 3BL, UK
}

Over the course of the last 2 decades the field of shoulder has greatly expanded, both in terms of elective, and trauma surgery. Developments in implant manufacture and bearing surfaces have allowed the development of a range of arthroplasty systems, allowing total and and partial shoulder replacement surgery to progress from conception to the reliable range of procedures and implants we now see.

Degenerate shoulder disease is becoming a more common problem for clinicians in the UK and throughout the world, with osteoarthritis one of the most common presenting complaints to shoulder surgeons. Furthermore, rheumatoid disease remains a widespread problem, and the use of shoulder replacement surgery, reverse shoulder replacement, and partial replacement surgery such as hemiresurfacing and hemiarthroplasty of the shoulder have become valuable treatment options for these conditions. This hot topic issue contains two articles summarising the indications for, technicalities of, and clinical outcomes of total and reverse arthroplasty of the shoulder, and hemiarthroplasty and hemiresurfacing, giving a brief overview of the technology and the technicalities of the procedures.

In addition to reviews relating to surgery for degenerate and inflammatory shoulder disease, this editorial contains several articles describing the pathology and treatment for common shoulder conditions. For instance, shoulder impingement syndrome is comprehensively reviewed, bringing together a summary of the recent literature relating

*Address correspondence to this author at the Department of Trauma and Orthopaedics, Macclesfield District General Hospital, East Cheshire NHS Trust, Victoria Road, Macclesfield, SK10 3BL, UK;

Tel: 01625 661307; Fax: 01625 661436; E-mail: was33uk@yahoo.com

${ }^{\S}$ Guest Editors. to the treatment of this common but often disabling condition. Adhesive capsulitis is discussed in depth in an article which describes the common misconceptions of the pathophysiology and natural history of the condition, and also describes the evidence base for the common treatment modalities.

Shoulder trauma is covered by several articles in this hot topic issue. Principally discussed in the article on proximal humerus fractures, bony trauma to the shoulder has been treated with increasing success in recent years with the advent of locking plate technology and the development of reverse shoulder arthroplasty, which is a valuable alternative to traditional acute hemiarthroplasty procedures used for complex fracture and fracture-dislocations of the shoulder. Furthermore, the review article on outcomes of treatment for fractures of the clavicle draws together the evidence base which has greatly expanded for the surgical and non surgical treatment of these injuries.

Describing the more clinical aspects of elective and acute shoulder surgery, two articles discuss the clinical assessment of the shoulder and the considerations for pre-and peri operative care. Complimenting the latter of these, an overview of anaesthesia in shoulder surgery is included. These articles will enable both newly trained and experience shoulder surgeons and their allied specialist colleagues to update themselves on recent evidence and trends on the subject.

Finally, adding some historical basis and giving a more modern context to this hot topic issue, are two articles discussing the history of shoulder surgery and future concepts. We hope that this issue provides and illuminating account of the present understanding of common shoulder pathology, the clinical aspects, and the current trends in various different treatment modalities.

(C) MacFarlane and Waseem; Licensee Bentham Open.

This is an open access article licensed under the terms of the Creative Commons Attribution Non-Commercial License (http://creativecommons.org/licenses/by-nc/3.0/) which permits unrestricted, non-commercial use, distribution and reproduction in any medium, provided the work is properly cited. 